\title{
Cannabinoid receptor 2 deficiency enhances isoflurane-induced spatial cognitive impairment in adult mice by affecting neuroinflammation, neurogenesis and neuroplasticity
}

\author{
$\mathrm{CHAO} \mathrm{LI}^{1 *}$, JINGPU SHI $^{1 *}$, JIAGUANG SUN $^{2}$, YUANYUAN SHI $^{1}$ and HUIQUN JIA ${ }^{1}$ \\ ${ }^{1}$ Department of Anesthesiology, The Fourth Hospital of Hebei Medical University, Shijiazhuang, Hebei 050011; \\ ${ }^{2}$ Department of Anesthesiology, Xingtai People's Hospital, Xintai, Hebei 054001, P.R. China
}

Received February 12, 2021; Accepted May 21, 2021

DOI: $10.3892 / \mathrm{etm} .2021 .10340$

\begin{abstract}
Isoflurane (Iso) is a commonly used inhalational anesthetic and is associated with the incidence of postoperative cognitive dysfunction (POCD). Cannabinoid receptor 2 (CB2R) was previously reported to have a promising neuroprotective function in cases of POCD, but the specific mechanisms have remained to be fully explored. The aim of the present study was to investigate the effect of CB2R deficiency on spatial cognitive performance in adult mice exposed to Iso. A total of 20 adult CB2R knockout (KO) and 20 wildtype (WT) mice were exposed to Iso (1.4\% in oxygen for $4 \mathrm{~h}$ ) or $100 \%$ oxygen. The Morris water maze (MWZ) test was performed 10 days after Iso exposure. Immunofluorescence staining and reverse transcription-quantitative PCR were performed to assess the expression of microglial marker ionized calcium-binding adaptor molecule-1 (Iba1) and the mRNA expression levels of microglial phenotype markers (M1: Interleukin-6, tumor necrosis factor- $\alpha$, inducible nitric oxide synthase; M2: Chitinase-3 like protein) in the hippocampus. Changes in hippocampal neurogenesis and neuroplasticity were assessed by 5-bromodeoxyuridine (BrdU) immunostaining and Golgi staining. Compared with
\end{abstract}

Correspondence to: Dr Huiqun Jia, Department of Anesthesiology, The Fourth Hospital of Hebei Medical University, 12th Health Road, Shijiazhuang, Hebei 050011, P.R. China

E-mail: jysyjiahuiqun@163.com

*Contributed equally

Abbreviations: ANOVA, analysis of variance; BrdU, 5-bromodeoxyuridine; CB2R, cannabinoid receptor 2; DG, dentate gyrus; Iba1, ionized calcium-binding adaptor molecule-1; IL-6, interleukin-6; iNOS, inducible nitric oxide synthase; Iso, isoflurane; $\mathrm{KO}$, knockout; LTP, long-term potentiation; MWZ, Morris water maze; POCD, postoperative cognitive dysfunction; TNF- $\alpha$, tumor necrosis factor- $\alpha$; WT, wild-type; Ym1/2, chitinase-3 like protein

Key words: cannabinoid receptor 2, isoflurane, cognition, neuroinflammation, neurogenesis, neuroplasticity control mice, WT Iso-exposed mice had impaired spatial performance in the MWZ test. Furthermore, hippocampal Iba1 immunoreactivity and the number of microglial branches were notably increased in Iso-exposed WT mice. This was paralleled by significant upregulation of M1-associated markers and downregulation of M2-associated markers in the hippocampus. An obviously reduced number of $\mathrm{BrdU}^{+}$ neurons and decreased spine density were observed in WT Iso-exposed mice compared with control mice. Of note, CB2R deficiency exacerbated the spatial cognition impairment induced by Iso in the MWZ test. The alterations in the activation, morphology and M1 polarization of microglia, the number of $\mathrm{BrdU}^{+}$neurons and spine density were more pronounced in $\mathrm{CB} 2 \mathrm{R}$-deficient Iso-exposed $\mathrm{KO}$ mice than in WT Iso-exposed mice. These results suggested that CB2R has a crucial role in Iso-induced cognitive impairment, which may be related to changes in hippocampal neuroinflammation, neurogenesis and neuroplasticity.

\section{Introduction}

Postoperative cognitive dysfunction (POCD) is one of the most common and devastating complications in patients undergoing major surgery under general anesthesia (1). Patients with POCD generally present with a striking decline in cognitive abilities, including memory, concentration, attention and cognitive flexibility (2). Previous clinical studies have indicated that POCD is closely associated with elevated healthcare costs, extended hospitalization and increased mortality (3). While factors such as advanced age, pre-existing cognitive impairment, lengthy surgery and general anesthesia have been established as the major risk factors for POCD (4), the pathogenesis and mechanisms remain somewhat elusive.

An accumulating body of evidence suggests that POCD shares a significant mechanical connection and commonality with Alzheimer's disease, in which altered microglia and astrocytic cells release extensive proinflammatory cytokines, resulting in persistent neuroinflammation and synaptic impairment (5). The various inflammatory components of neuroinflammation, including proinflammatory cytokines, chemokines and glial cells, have a fundamental role in regulating the neurogenesis process and dendric spine density in 
cognition-related brain regions, such as the hippocampus $(6,7)$. Multiple perioperative factors, including surgical stimulation and inhaled anesthetics, may accelerate this process by enhancing proinflammatory factors. Overproduction of these proinflammatory molecules results in a negative and toxic response in neurons, including dysfunction in neurogenesis, neural plasticity and long-term potentiation (LTP), eventually leading to cognitive decline (8). Thus, surgery/ anesthesia-induced neuroinflammation has been indicated to promote the incidence and development of POCD and candidate interventions targeting aberrant neuroinflammation have aroused global interest (9). Microglia are considered as the innate immunity cells in the central nervous system and their activation and M1/M2 polarization are crucial to the process of neuroinflammation (10). Furthermore, the physiological functions of microglia in neurogenesis and synaptic plasticity may be dampened in neurodegenerative diseases (11). Regulating the activation and M1/M2 polarization of microglia in neuroinflammation may have a potential therapeutic effect on cognitive ability in neurodegenerative diseases and POCD.

Cannabinoid receptor 2 (CB2R) is a crucial neuromodulatory target in the central nervous system and has an important role in homeostatic control and numerous neurodegenerative diseases. CB2R is reported to be expressed in both microglia and neurons in healthy brains at low levels and is markedly upregulated by microglia during pathological conditions. Previous studies have demonstrated the vital role of CB2R in microglial activity and polarization in the regulation of neuroinflammatory processes (12). Accumulated evidence suggests a critical role of $\mathrm{CB} 2 \mathrm{R}$ in mediating neuroinflammation, neurogenesis and neuroplasticity, and it is a promising pharmacological target for Alzheimer's disease and POCD $(13,14)$. Pharmacological intervention or genetic deletion of CB2R may affect the pathological development of Alzheimer's disease in model mice, accompanied by attenuation of neuroinflammation and improvement of cognitive impairment $(15,16)$. Another study indicated that activation of CB2R may effectively attenuate surgery/isoflurane (Iso)-induced spatial memory impairment by downregulating hippocampal microglial activation and pro-inflammatory factors (17). Bromodeoxyuridine (BrdU) immunohistochemistry has been applied for the study of neurogenesis in the adult mammalian brain (18) and activation of CB2R was also reported to significantly increase the $\mathrm{BrdU}^{+}$cells in a mouse model of stroke (19). Golgi staining is considered as a classical method to detect dendritic spine (20) and activation of CB2R by minocycline increased the number of dendritic spines in the hippocampus and enhanced learning and memory of aged mice (21). However, the mechanisms underlying the therapeutic effect of CB2R on Iso-induced POCD model mice have not been extensively investigated. Thus, the present study was performed to investigate the effects of CB2R deficiency on spatial memory, hippocampal neuroinflammation, neurogenesis and neuroplasticity in mice with Iso-induced POCD.

\section{Materials and methods}

Animals. Five female CB2R knockout (KO) mice (aged 3 months; weight, 25-30 g) were purchased from the Jackson
Laboratory and originally created on the background of $\mathrm{C} 57 \mathrm{BL} / 6 \mathrm{~J}$ mice. Based on the protocol of a previous study (22), female CB2R KO mice were crossed with five male wild-type (WT) C57BL/6J mice (aged 3 months; weight, 25-30 g; purchased from Sipeifu Biotechnology Co.) to obtain heterozygous $\mathrm{CB} 2 \mathrm{R}^{+/-}$mice, and then the $\mathrm{CB} 2 \mathrm{R}^{+/}$mice were bred with each other to generate littermates of $\mathrm{CB}_{2} \mathrm{R}^{+/+}(\mathrm{CB} 2 \mathrm{R}$ WT) and $\mathrm{CB} 2 \mathrm{R}^{-/-}(\mathrm{CB} 2 \mathrm{R} \mathrm{KO})$. All mice were group-housed in a standard rodent unit with free access to food and water. All mice were genotyped by PCR using the following primers: CB2R KO, 5'-GGGGATCGATCCGTCCTGTAAGTCT-3'; CB2R WT, (5'-GGAGTTCAACCCCATGAAGGAGTAC-3'); and CB2R common (5'-GACTAGAGCTTTGTAAGGCGG G-3') (23). 'Common' refers to primers used for both experimental types of mice. Primers for the CB2R KO mice included CB2R KO and CB2R common, and primers for the CB2R WT mice included CB2R WT and CB2R common.

All experiments were performed with the approval of the Animal Care Committee of the Fourth Hospital of Hebei Medical University (Shijiazhuang, China).

Animal groups and treatment. A total of $20 \mathrm{CB} 2 \mathrm{R}$ KO mice and 20 WT littermates (CB2R WT) (aged 3 months; weight, 25-30 g) were randomly divided into groups $(n=10$ in each group): $\mathrm{CB} 2 \mathrm{R} \mathrm{WT}+\mathrm{O}_{2}, \mathrm{CB} 2 \mathrm{R} \mathrm{KO}+\mathrm{O}_{2}, \mathrm{CB} 2 \mathrm{R} \mathrm{WT}+\mathrm{Iso}$ and $\mathrm{CB} 2 \mathrm{R} \mathrm{KO}+\mathrm{Iso}$. The level of Iso exposure was based on previous reports indicating that $1.4 \%$ Iso for $>4 \mathrm{~h}$ resulted in cognitive impairment in young adult C57BL/6 mice (23). The mice from the CB2R WT+Iso and CB2R KO+Iso groups received $1.4 \%$ Iso in $100 \%$ oxygen for $4 \mathrm{~h}$ using a rodent anesthesia machine (R610; RWD Life Science Co., Ltd.) based on previous literature (24). The mice from the $\mathrm{CB} 2 \mathrm{R} \mathrm{WT}+\mathrm{O}_{2}$ and $\mathrm{CB} 2 \mathrm{R} \mathrm{KO}+\mathrm{O}_{2}$ groups received $100 \%$ oxygen for $4 \mathrm{~h}$ and the temperature was maintained at $37 \pm 0.5^{\circ} \mathrm{C}$ using a heating pad during Iso treatment.

Morris water maze (MWZ) test. After a 10 day washing period between Iso treatment and examination based on previous literature (24), the MWZ test was performed to evaluate the spatial learning and memory capabilities of the mice, as previously reported $(25,26)$. An open-field circular pool was filled with warm water $\left(23^{\circ} \mathrm{C}\right)$ to a height of $30 \mathrm{~cm}$ and a white hidden platform (10 cm in diameter) was submerged $1.5 \mathrm{~cm}$ below the water surface. A black curtain separated the pool from its surroundings in an isolated room. An automated video tracking system recorded the motion of each mouse and the data were analyzed using MWZ test analysis software (version: DigBehv-MM; Jiliang Co.). During the five consecutive training days, each mouse performed four trials of the navigation test daily. The escape latency to the hidden platform and swimming speed were recorded. On the sixth day, the hidden platform was removed and the number of times the former location of the platform was crossed was recorded.

After the cognitive performance experiments, mice were injected with a dose of sodium pentobarbital $(50 \mathrm{mg} / \mathrm{kg}$, intraperitoneally) and sacrificed by cervical dislocation. Their brains were removed from skulls and rapidly dissected into two hemispheres. One hemisphere was post-fixed in $4 \%$ paraformaldehyde for $24 \mathrm{~h}$, incubated in $30 \%$ sucrose for $12 \mathrm{~h}$ and then processed for immunofluorescence staining and BrdU 
immunostaining. The other hemisphere was frozen immediately on dry ice, stored at $-80^{\circ} \mathrm{C}$ and processed for RNA extraction.

Immunofluorescence staining. Immunofluorescence staining of the hippocampal slices was performed as described in a previous study by our group (15). All hippocampal sections were incubated with $3 \%$ bovine serum albumin (OriGene Technologies, Inc.) for $20 \mathrm{~min}$ at room temperature $\left(25-30^{\circ} \mathrm{C}\right)$ to block non-specific binding sites. The sections were then incubated with the primary antibody, rabbit polyclonal anti-ionized calcium-binding adaptor molecule-1 (Iba1; cat. no. ab153696; 1:500; Abcam), for $12 \mathrm{~h}$ at $4^{\circ} \mathrm{C}$. The sections were then incubated with Alexa Fluor 488-conjugated secondary antibodies (cat. no. ab150077; 1:100; Abcam) for $2 \mathrm{~h}$ at room temperature $\left(25-30^{\circ} \mathrm{C}\right)$, followed by counterstaining with DAPI. Representative fluorescence microscopy images of the hippocampal samples were taken and immunofluorescence staining intensity was quantified using a Nikon Eclipse Ni-U microscope (Nikon Corporation) at x10 magnification and image pro plus software version 6.0 (Media Cybernetics, Inc.) by an investigator who was blinded to the origin of the sections. Integrated optical density of each slice from each group was measured and presented as a percentage with $\mathrm{CB} 2 \mathrm{R} \mathrm{WT}+\mathrm{O}_{2}$ as the control. Microglial morphology was analyzed using Imaris software (version 8.1; Oxford Instruments) at x100 magnification to assess the number of microglial branches.

RNA extraction and reverse transcription-quantitative $P C R(R T-q P C R)$ analysis. Total RNA was extracted from the hippocampal tissue using the RNeasy mini kit (Qiagen $\mathrm{GmbH}$ ) following the manufacturer's protocol. RT to generate cDNA was performed using the ReverTra Ace qPCR RT Master Mix with gDNA Remover kit (Toyobo Co., Ltd.) according to the manufacturer's protocol. qPCR analysis was performed using the FastStart Essential DNA Green Master kit (Roche) according to the manufacturer's protocol. The primer sequences of the target genes were as follows: Interleukin-6 (IL-6), 5'-TGCAAGAGACTTCCATCCAGTT-3' (forward) and 5'-GAAGTAGGGAAGGCCGTGG-3'(reverse); tumornecrosis factor- $\alpha$ (TNF- $\alpha), 5$ '-GCACCACCATCAAGGACTC-3' (forward) and 5'-TGAGACAGAGGCAACCTGAC-3' (reverse); inducible nitric oxide synthase (iNOS), 5'-GGCAGCCTGTGAGACCTTTG-3' (forward) and 5'-GCATTGGAAGTGAAGCGTTTC-3' (reverse); chitinase-3 like protein (Ym1/2), 5'-CAGGGTAATGAGTGGGTTGG-3' (forward) and 5'-CACGGCACCTCCTAAATTGT-3' (reverse); GAPDH, 5'-ACTCCACTCACGGCAAATTC-3' (forward) and 5'-TCTCCATGGTGGTGAAGACA-3' (reverse). The PCR amplification conditions were adjusted based on the manufacturer's protocol and previous research (15): $95^{\circ} \mathrm{C}$ for $10 \mathrm{~min}$ and 45 cycles of $95^{\circ} \mathrm{C}$ for $10 \mathrm{sec}$ and $60^{\circ} \mathrm{C}$ for $1 \mathrm{~min}$. The expression levels of these genes were normalized to the levels of GAPDH and presented as $2^{-\Delta \Delta \mathrm{Cq}}$ with $\mathrm{CB} 2 \mathrm{R} \mathrm{WT}+\mathrm{O}_{2}$ as the control (27).

BrdU injection and immunostaining. All mice received an intraperitoneal injection of BrdU (Sigma-Aldrich; Merck $\mathrm{KGaA}$ ) at a dose of $50 \mathrm{mg} / \mathrm{kg}$ twice a day for five consecutive days and were sacrificed $24 \mathrm{~h}$ after the last BrdU injection (28).
For BrdU staining, coronal sections were incubated in $2 \mathrm{~N}$ $\mathrm{HCl}$ at $37^{\circ} \mathrm{C}$ for $30 \mathrm{~min}$ to denature the DNA. The sections were then incubated with $1 \%$ bovine serum albumin (cat. no. ZLI-9027; OriGene Technologies, Inc.) for $30 \mathrm{~min}$ at room temperature $\left(25-30^{\circ} \mathrm{C}\right)$, followed by incubation with anti-BrdU antibody (cat. no. 6326; 1:500 dilution; Abcam) at $4^{\circ} \mathrm{C}$ for $12 \mathrm{~h}$. The primary antibody was detected by subsequent incubation with the corresponding biotinylated secondary antibody (cat. no. ZB-2040; 1:100; OriGene Technologies, Inc.) and a diaminobenzidine kit (cat. no. ZLI-9017; OriGene Technologies, Inc.) at room temperature $\left(25-30^{\circ} \mathrm{C}\right)$ for $1 \mathrm{~h}$. Stained sections were imaged by an investigator blinded to the treatment conditions using an Olympus VS120 light microscope at $\mathrm{x} 4$ magnification (Olympus Corporation). All BrdU' cells in the dentate gyrus (DG) of the hippocampus were identified and counted by an investigator who was blinded to the origin of the section. The average numbers of $\mathrm{BrdU}^{+}$cells in three sections per mouse were analyzed.

Golgi staining and spine density analysis. Golgi staining was performed according to the protocol of the FD Rapid Golgistain $^{\text {TM }}$ kit (FD NeuroTechnologies, Inc.), as previously described (15). Mouse brain tissues were impregnated with solutions A and B for 2 weeks (room temperature, in the dark), followed by incubation in solution $\mathrm{C}$ for 1 week $\left(4^{\circ} \mathrm{C}\right.$, in the dark). Next, brain tissues were cut into $100-\mu \mathrm{m}$ coronal sections. Sections containing the hippocampus were stained with solutions D and E mixed with distilled water and dehydrated in a graded ethanol series. Images were acquired using an Olympus IX-81 microscope (Olympus Corporation) with a $60 x$ oil lens. Dendritic spine density in the DG of the hippocampus was measured using Imaris software (version 8.1; Oxford Instruments) in a blinded manner.

Statistical analysis. All statistical analyses were performed using GraphPad Prism version 7.0 (GraphPad Software, Inc.). Values are expressed as the mean \pm standard error of mean. Results of the escape latencies and swimming speeds from the MWZ tests were analyzed using two-way repeated-measures ANOVA followed by Tukey's post-hoc test. For other data, a two-way ANOVA was performed, if appropriate. $\mathrm{P}<0.05$ was considered to indicate a statistically significant difference.

\section{Results}

CB2R deficiency aggravates Iso-induced spatial cognitive impairment in adult mice. The timeline of the experimental procedures is presented in Fig. 1A. To examine whether CB2R deficiency affected spatial learning and memory in the Iso-induced POCD mouse model, animals were subjected to MWZ tests. It was revealed that Iso exposure and CB2R deficiency did not affect the swimming speed on the five training days (Fig. 1B). The results indicated that the CB2R WT+Iso group had a significantly longer escape latency and a smaller number of platform crossings than the $\mathrm{CB} 2 \mathrm{R} \mathrm{WT}+\mathrm{O}_{2}$ group $(\mathrm{P}<0.05$ and $\mathrm{P}<0.001$, respectively; Fig. $1 \mathrm{C}$ and $\mathrm{D})$. Furthermore, CB2R KO+Iso mice had a significantly longer escape latency and a smaller number of platform crossings than the $\mathrm{CB} 2 \mathrm{R}$ WT+Iso group $(\mathrm{P}<0.05$ and $\mathrm{P}<0.05$, respectively; Fig. $1 C$ and D). Representative swimming paths on the sixth 
A

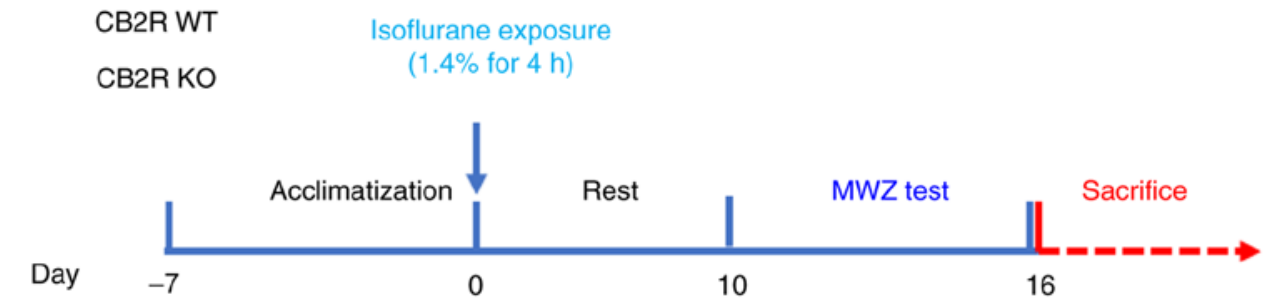

B

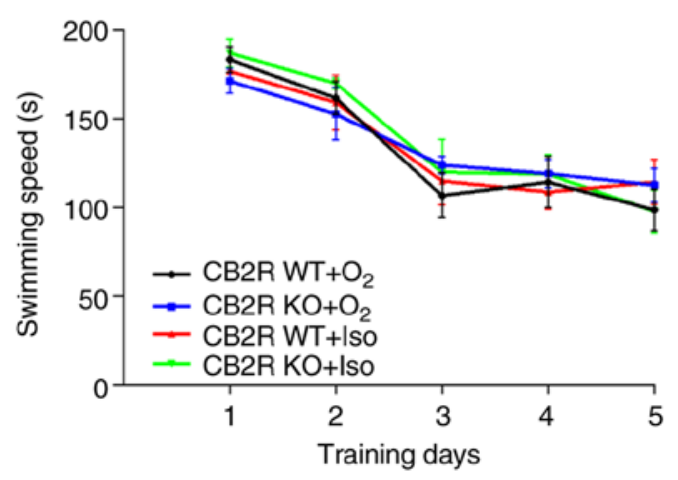

D

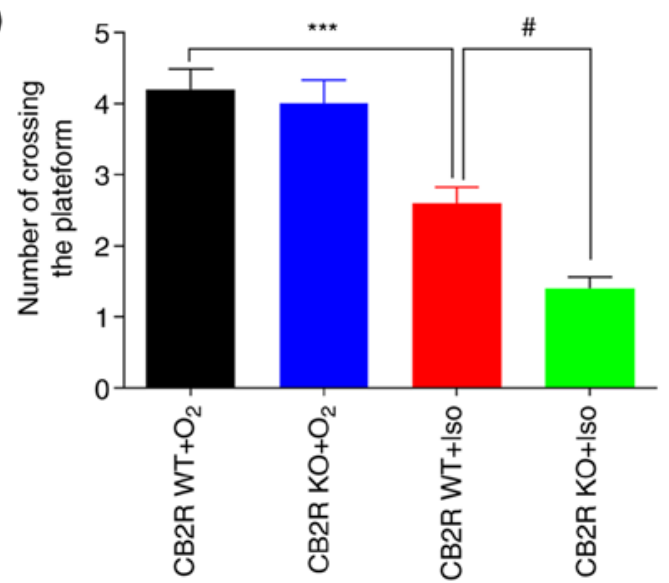

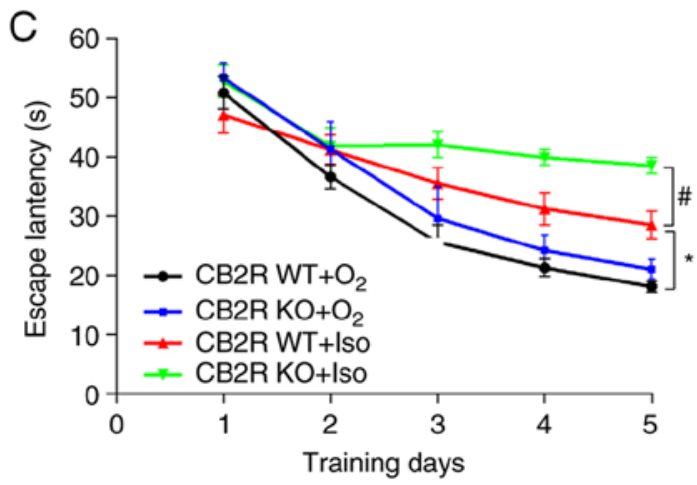

E

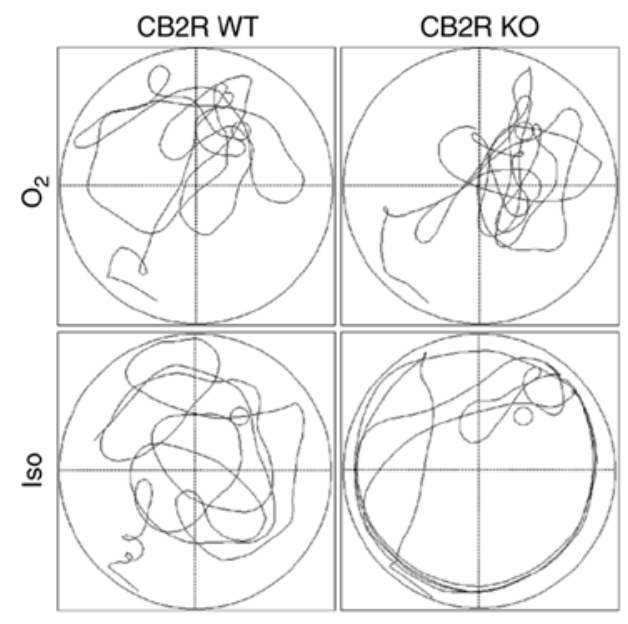

Figure 1. CB2R deficiency enhances Iso-induced spatial memory impairment in the MWZ test. (A) Schematic diagram of the experimental procedures. (B) Swimming speed. (C) Mean escape latency. (D) Crossing the platform. (E) Representative path tracing on the sixth day of the MWZ test. Values are expressed as the mean \pm standard error of the mean $(n=10)$. Results of escape latency and swimming speed were assessed by two-way repeated-measures ANOVA and other statistical comparisons were performed by two-way ANOVA. ${ }^{*} \mathrm{P}<0.05,{ }^{* * *} \mathrm{P}<0.001$ and ${ }^{*} \mathrm{P}<0.05$. CB2R, cannabinoid receptor 2 ; MWZ, Morris water maze; WT, wild-type; KO, knockout; sec, seconds; Iso, isoflurane.

day are provided in Fig. 1E. The present results indicated that CB2R deficiency aggravated the spatial learning and memory deficits induced by Iso exposure.

CB2R deficiency enhances Iso-induced microglial activation and branching in the hippocampus of adult mice. To investigate whether $\mathrm{CB} 2 \mathrm{R}$ deficiency affected microglial activation and morphology in the Iso-induced POCD model mice, microglia were subjected to immunofluorescence staining for Iba1, an efficient and specific microglial biomarker (29). The results indicated increased Iba1 immunoreactivity and a larger number of microglial branches in the hippocampus of the CB2R WT+Iso group compared to the CB2R WT+O group $(\mathrm{P}<0.01$; Fig. 2A-D). In addition, Iba1 immunoreactivity and the number of microglial branches in the hippocampus of the CB2R KO+Iso group were significantly increased in comparison with the CB2R WT+Iso group $(\mathrm{P}<0.05$; Fig. 2A-D). These results demonstrated that CB2R deficiency facilitated Iso-induced microglial activation and branching in the hippocampus of adult mice.

CB2 receptor deficiency promotes microglial $M 1$ polarization in the hippocampus of adult mice exposed to Iso. In general, in numerous neurodegenerative diseases, activated microglia are driven to polarize to two opposite subtypes: M1 phenotype expressing the markers IL-6, TNF- $\alpha$ and iNOS or the M2 phenotype characterized by $\mathrm{Ym} 1 / 2$ expression (10). To explore the effect of CB2R deficiency on microglial M1/M2 polarization in an Iso-induced mouse model of POCD, RT-qPCR analysis was performed to evaluate the mRNA expression levels of IL-6, TNF- $\alpha$, iNOS and Ym1/2. The results indicated significant upregulation of M1-associated mRNA levels and 
A

A

CB2R WT

CB2R KO

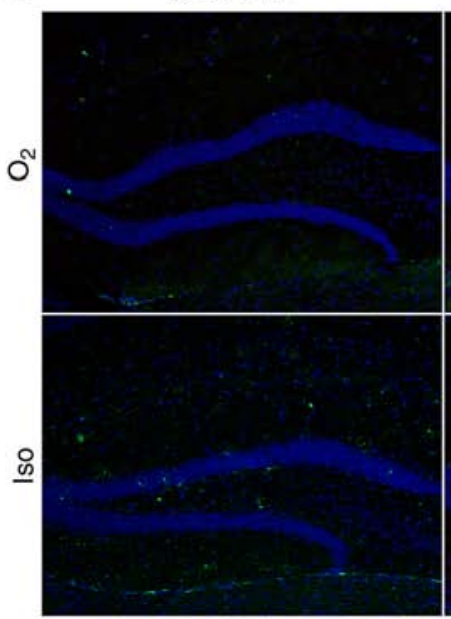

C

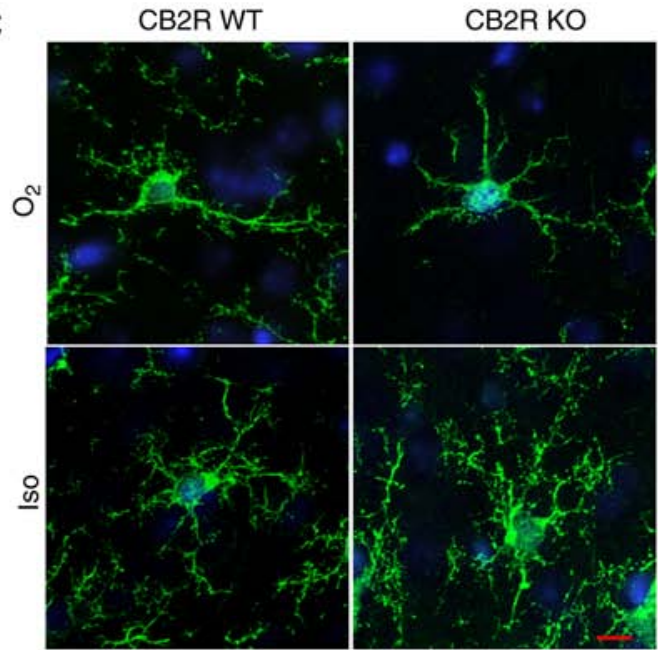

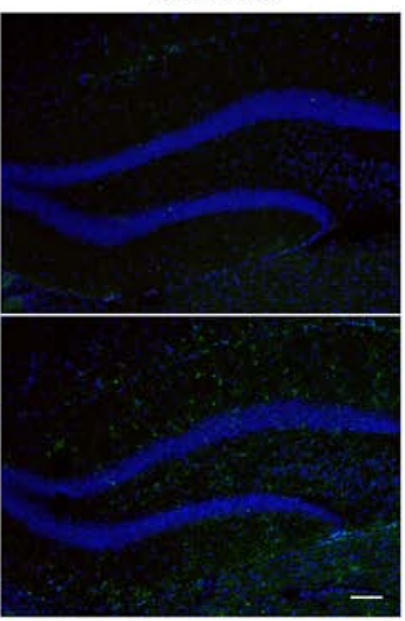

CB2R KO
B
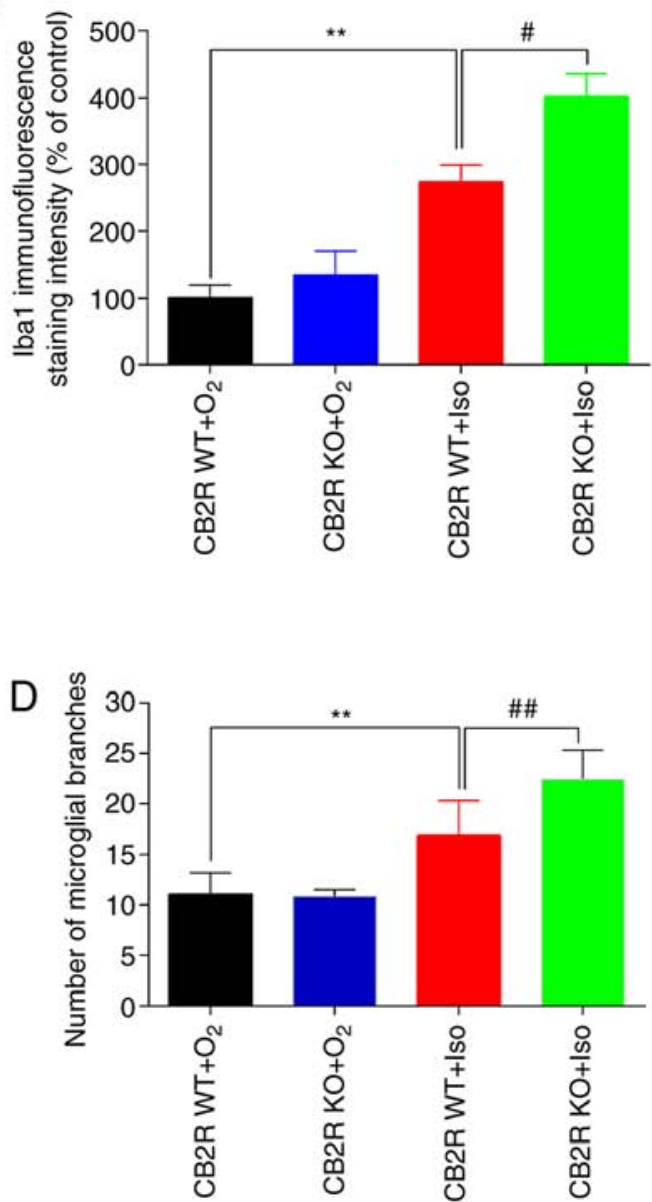

Figure 2. CB2R deficiency enhances Iso-induced activation and affects the morphology of microglia in the hippocampus of adult mice. (A) Representative images of immunofluorescence staining for Iba1 (green) and nuclei stained in blue (white scale bar, $100 \mu \mathrm{m}$ ). (B) Quantification of Iba1 immunofluorescence staining intensity in the hippocampus. (C) Representative images of Iba1 (green) and nuclei (blue) by immunofluorescence staining (red scale bar, $10 \mu \mathrm{m}$ ). (D) Number of Iba1+ microglial branches in the hippocampus. Values are expressed as the mean \pm standard error of the mean ( $\mathrm{n}=6$ ) and analyzed using two-way ANOVA, ${ }^{* *} \mathrm{P}<0.01,{ }^{\#} \mathrm{P}<0.05$ and ${ }^{\# \#} \mathrm{P}<0.01$. CB2R, cannabinoid receptor 2 ; Iba1, ionized calcium-binding adaptor molecule-1; WT, wild-type; KO, knockout; Iso, isoflurane.

downregulated M2-associated mRNA levels in the CB2R WT+Iso group compared with the $\mathrm{CB} 2 \mathrm{R}$ WT$+\mathrm{O}_{2}$ group (all $\mathrm{P}<0.05$; Fig. 3A-D). Of note, these changes in microglial $\mathrm{M} 2 / \mathrm{M} 1$ polarization in the $\mathrm{CB} 2 \mathrm{R} \mathrm{WT}+\mathrm{Iso}$ group were greater than those in the CB2R KO+Iso group (all $\mathrm{P}<0.05$; Fig. 3A-D). Thus, CB2R deficiency significantly influenced Iso-induced microglial polarization by promoting M1 and suppressing M2 phenotype expression.

CB2 receptor deficiency aggravates neurogenesis damage in the hippocampus of adult mice exposed to Iso. Inhibition of hippocampal neurogenesis has been demonstrated to be a vital hallmark of the POCD model (30). To investigate the effect of CB2R deficiency on neurogenesis in an Iso-induced POCD mouse model, BrdU immunostaining was performed to label proliferating neural progenitor cells. The results revealed that CB2R WT+Iso mice had significantly fewer BrdU ${ }^{+}$cells in the hippocampus compared with the $\mathrm{CB} 2 \mathrm{R} \mathrm{WT}+\mathrm{O}_{2}$ group $\left(\mathrm{P}<0.001\right.$; Fig. 4A and B). Furthermore, the number of $\mathrm{BrdU}^{+}$ cells in $\mathrm{CB} 2 \mathrm{R} \mathrm{KO}+\mathrm{Iso}$ mice was significantly lower than that in CB2R WT+Iso mice ( $\mathrm{P}<0.05$; Fig. $4 \mathrm{~A}$ and $\mathrm{B})$. These results indicated that $\mathrm{CB} 2 \mathrm{R}$ deficiency enhanced Iso-induced neurogenesis damage in the hippocampus of adult mice.

CB2 receptor deficiency reduces dendritic complexity in the hippocampus of adult mice exposed to Iso. Aberrant synaptic plasticity has been reported to be closely related to Iso-induced POCD (31). In Fig. 5A, Golgi staining demonstrated that neurons in the whole brain section from the control mice were interlaced, thus making neuronal changes in the whole brain section difficult to analyze. Thus, Golgi staining was performed to detect the spine density in the hippocampal DG region in the different groups. The results indicated that spine density in hippocampal DG neurons of CB2R WT+Iso mice was significantly lower than that of $\mathrm{CB} 2 \mathrm{R} \mathrm{WT}+\mathrm{O}_{2}$ mice $(\mathrm{P}<0.05$; Fig. 5B and $\mathrm{C})$. In addition, a marked reduction in spine density was determined in the hippocampal DG region of CB2R KO+Iso mice compared with that in CB2R WT+Iso mice $(\mathrm{P}<0.05$; Fig. $5 \mathrm{~B}$ and $\mathrm{C})$. These results indicated that $\mathrm{CB} 2 \mathrm{R}$ deficiency significantly enhanced the Iso-induced 
A

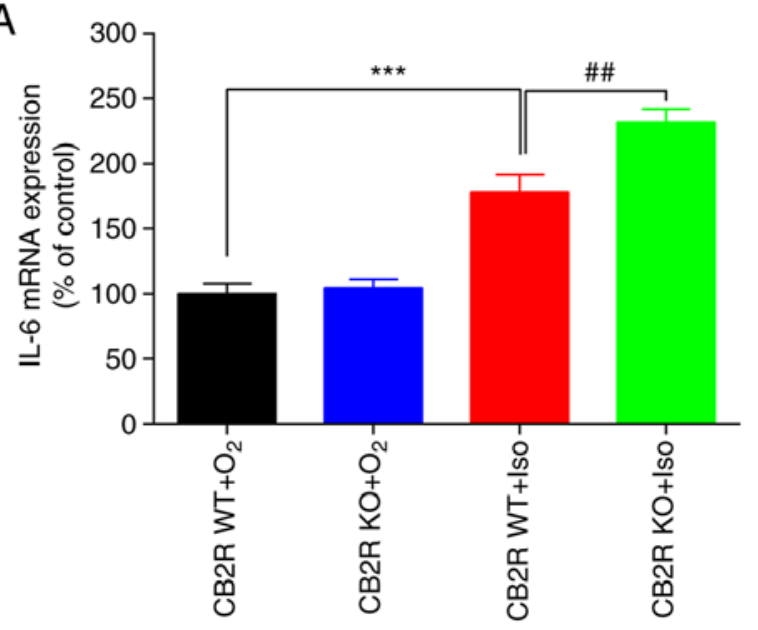

C

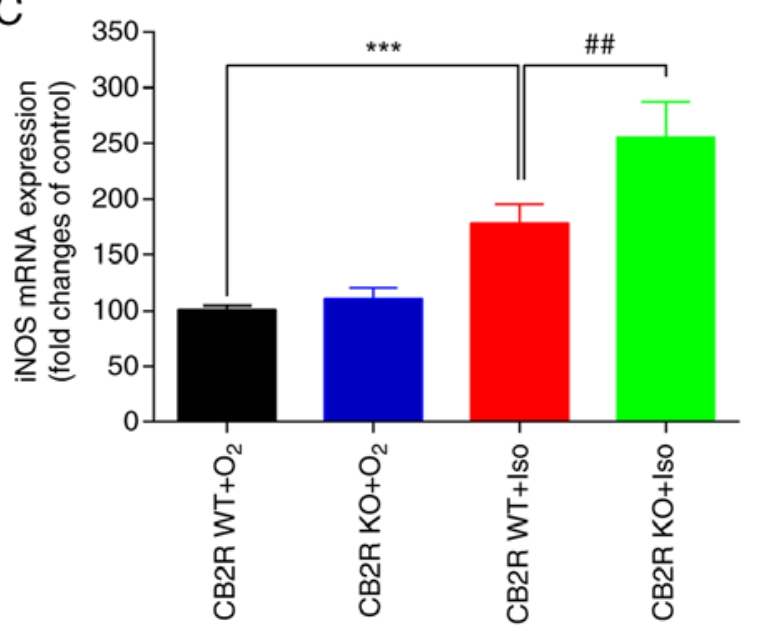

B
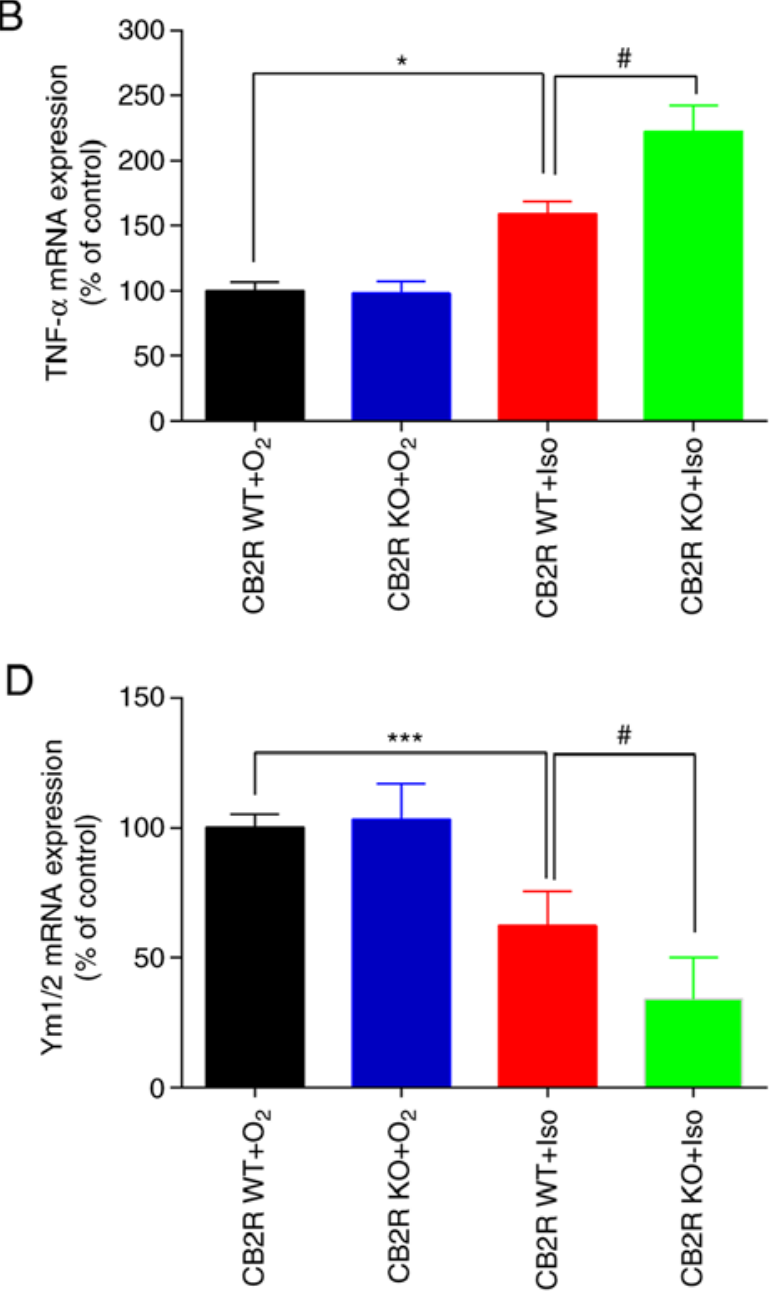

Figure 3. CB2R deficiency promotes microglial M1 polarization in the hippocampus of adult mice exposed to Iso. Quantification of the hippocampal mRNA levels of M1 microglia-associated biomarkers (A) IL-6; (B) TNF- $\alpha$; (C) iNOS; and (D) M2 microglia-associated marker Ym1/2. Values are expressed as the mean \pm standard error of the mean $(\mathrm{n}=6)$ and analyzed using two-way ANOVA. ${ }^{*} \mathrm{P}<0.05,{ }^{* * *} \mathrm{P}<0.001,{ }^{\#} \mathrm{P}<0.05$ and ${ }^{\# \#} \mathrm{P}<0.01$. CB2R, cannabinoid receptor 2 ; Iba1, ionized calcium-binding adaptor molecule-1; IL-6, interleukin-6; iNOS, inducible nitric oxide synthase; TNF- $\alpha$, tumor necrosis factor; Ym1/2, chitinase-3 like protein; Iso, isoflurane.

spine density reduction and dendritic complexity in the hippocampus of adult mice.

\section{Discussion}

POCD is one of the most urgent concerns for patients and clinicians worldwide, as it significantly increases medical costs, morbidity and mortality. Therefore, extensive research has been performed to investigate its underlying etiology and potential therapeutic targets. A large and growing body of evidence supports the theory that CB2R has a vital neuroprotective role in cognitive dysfunction diseases via modulation of microglia-associated neuroinflammation (32). The present study investigated the effects of CB2R deficiency on Iso-induced spatial cognition impairment in adult mice. The results indicated that CB2R KO mice with Iso exposure had a significantly poorer performance in the MWZ test, more pronounced changes in neuroinflammation parameters and more severe neurogenesis and neuroplasticity damage in the hippocampus than WT mice, indicating that CB2R deficiency made adult mice more vulnerable to the neurotoxicity of Iso, as summarized in the schematic in Fig. 6.

The MWZ test, a classical tool used to monitor hippocampus-related spatial learning and memory ability, has been widely applied in laboratory research (33). The results suggested that $\mathrm{CB} 2 \mathrm{R} \mathrm{KO}$ mice without Iso exposure had a similar cognitive score in the MWZ test compared with control mice and that Iso-exposed CB2R KO mice had a poorer performance in the MWZ test compared with Iso-exposed WT mice. However, previous studies on the effect of CB2R deficiency on cognition and memory were inconclusive. $\mathrm{Li}$ and $\mathrm{Kim}$ (23) reported that $\mathrm{CB} 2 \mathrm{R} \mathrm{KO}$ mice displayed impaired long-term contextual fear memory but enhanced spatial working memory. Schmöle et al (26) indicated that $\mathrm{CB} 2 \mathrm{R}$ deletion enhanced spatial learning of 6-month-old CB2R KO and APP/PS1 (double transgenic mice)/CB2R KO mice in the MWZ test. Aso et al (34) reported that $\mathrm{CB} 2 \mathrm{R}$ deficiency did not significantly affect memory impairment in a two-object recognition test of 3-month- and 6-month-old APP/PS1/CB2R KO mice. Of note, the present results also suggested that $\mathrm{CB} 2 \mathrm{R}$ deficiency 
A

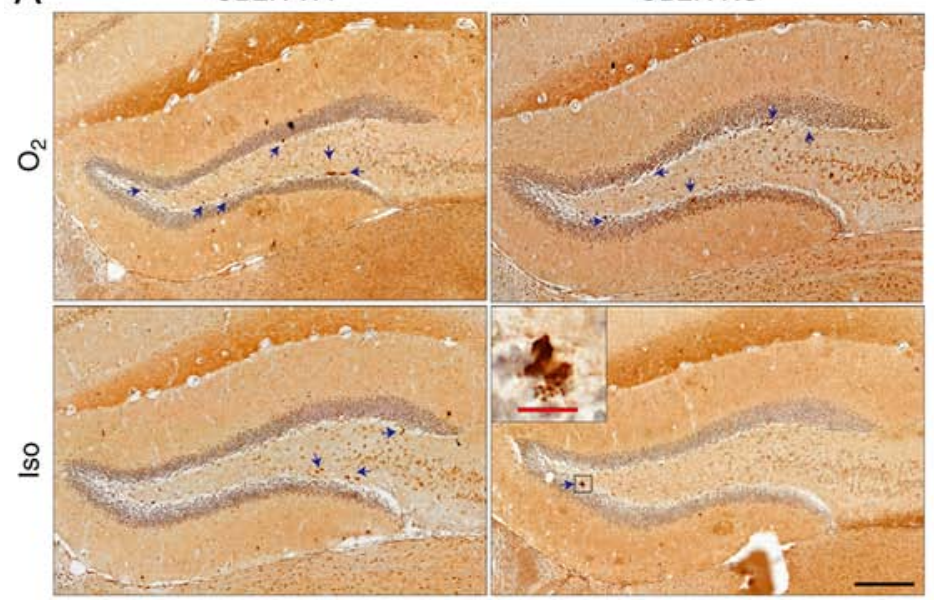

B

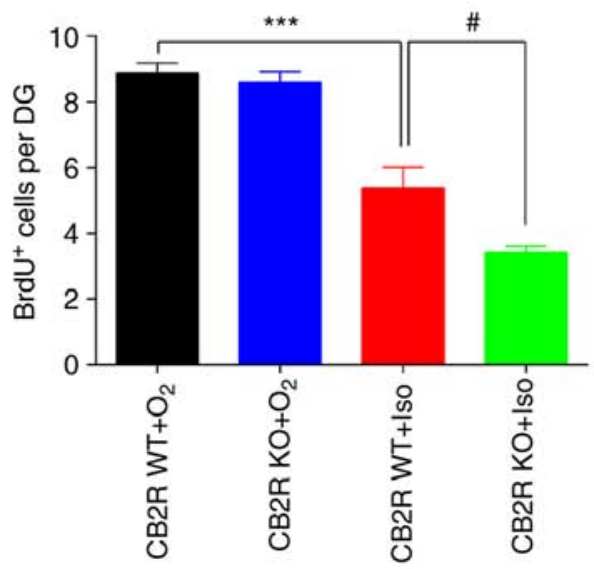

Figure 4. CB2R deficiency enhances neurogenetic damage in the hippocampus of adult mice exposed to Iso. (A) Representative images of immunostaining for BrdU (positive brown staining indicated with blue arrows; black scale bar, $200 \mu \mathrm{m}$; red scale bar in magnified window, $20 \mu \mathrm{m}$ ). (B) Quantification of the hippocampal BrdU ${ }^{+}$cells. Values are expressed as the mean \pm standard error of the mean $(\mathrm{n}=6)$ and analyzed using two-way ANOVA. ${ }^{* * *} \mathrm{P}<0.001$ and ${ }^{\#} \mathrm{P}<0.05$. BrdU, 5-bromodeoxyuridine; CB2R, cannabinoid receptor 2; WT, wild-type; KO, knockout; Iso, isoflurane; DG, dentate gyrus.

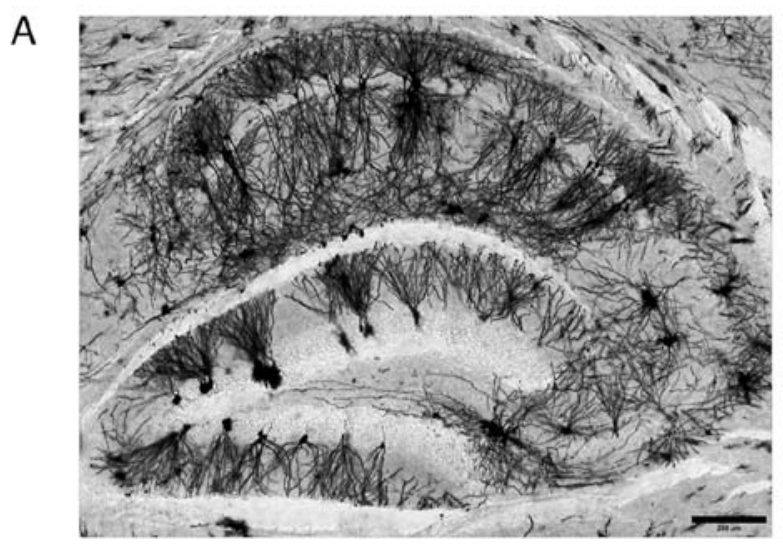

B

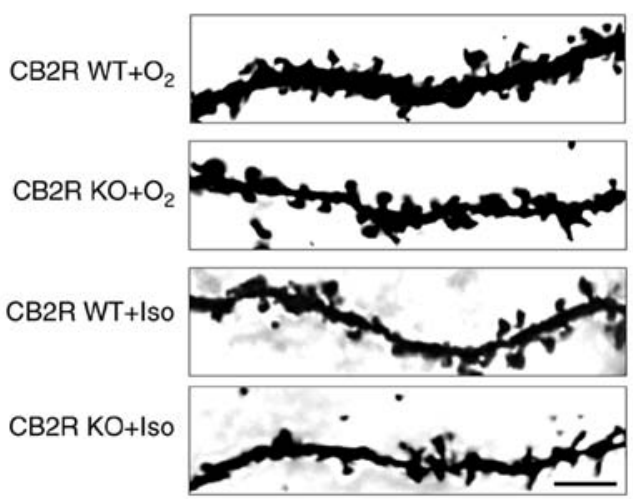

C

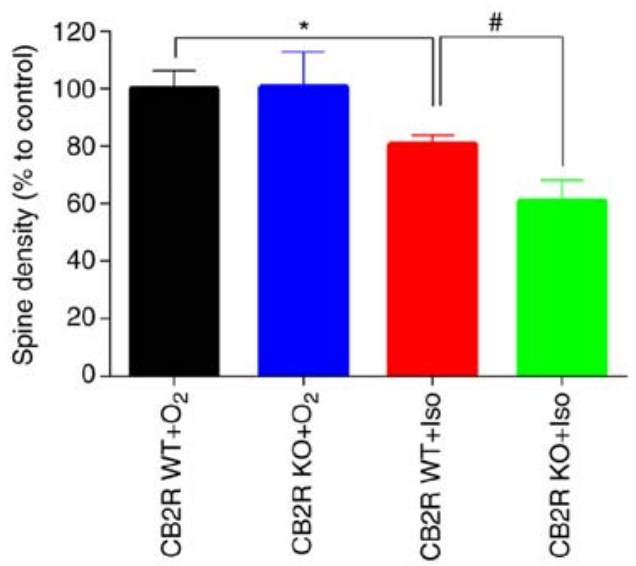

Figure 5. CB2R deficiency reduces dendritic complexity in the hippocampus of adult mice exposed to Iso. (A) A representative image of neurons with Golgi staining in the whole brain section (scale bar, $200 \mu \mathrm{m}$ ). (B) Representative images of hippocampal dendrite fragments with visible spines (scale bar, $5 \mu \mathrm{m}$ ). (C) Quantification of spine density in the hippocampal dentate gyrus. Values are expressed as the mean \pm standard error of the mean $(n=4)$ and analyzed using two-way ANOVA */\# $\mathrm{P}<0.05$. CB2R, cannabinoid receptor 2; WT, wild-type; KO, knockout; Iso, isoflurane.

in the absence of Iso exposure did not affect the cognitive performance. These independent studies imply that in the absence of Iso, CB2Rs alone have diverse, or even opposite roles, in regulating different types of cognition and memory, depending on the distinct disease stage. Moreover, combined surgery, anesthetic treatment or other injury factors and the effect of CB2 receptor deficiency on cognitive function requires further study. 


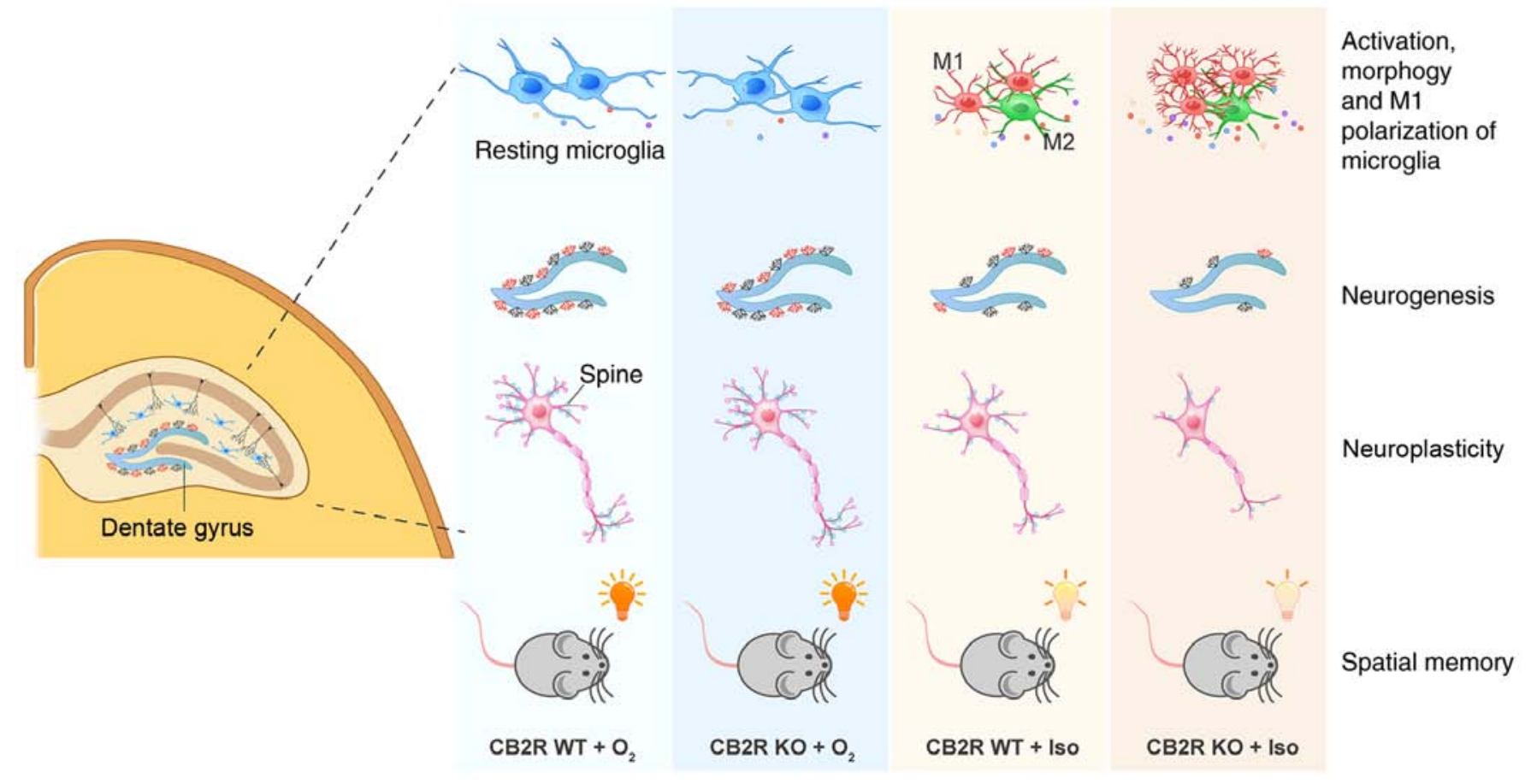

Figure 6. Schematic representation of the effects of CB2R deficiency on Iso-induced cognitive impairment. Iso exposure leads to spatial memory impairment of adult mice, accompanied by alterations in the activation, morphology and M1 polarization of microglia, BrdU ${ }^{+}$neurogenesis and spine density in the hippocampus. CB2R deficiency makes adult mice more vulnerable to Iso neurotoxicity, as indicated by more significant injury in terms of neuroinflammation, neurogenesis and neuroplasticity after Iso exposure. BrdU, 5-bromodeoxyuridine; CB2R, cannabinoid receptor 2; WT, wild-type; KO, knockout; Iso, isoflurane.

The neuroinflammatory hypothesis is considered one of the leading mechanisms of POCD, based on which various promising candidates for the prevention and treatment of POCD have been developed (9). Strategies targeting microglia to reduce the development of POCD may be feasible and viable (35). Iso exposure or surgery leads to post-operative cognitive dysfunction in aged rodents, accompanied by upregulated protein levels of pro-inflammatory cytokines TNF- $\alpha$, IL-1 $\beta$, interferon- $\gamma$ and microglia marker Iba- 1 in the hippocampus (36). Thus, immunofluorescence staining and RT-qPCR were performed in the present study to determine the effect of CB2R deficiency on microglial activity and mRNA expression of M1/M2 microglial phenotype markers in the whole hippocampus. The results indicated the presence of spatial cognitive impairment accompanied by significant alterations of activation, morphology and $\mathrm{M} 1$ polarization of microglia in the hippocampus of Iso-exposed CB2R WT mice compared with control mice. CB2R KO mice without Iso exposure had a similar expression of Iba1 and M1 polarization compared with control mice and Iso-exposed CB2R KO mice had more expression of Iba1 and M1 polarization compared with Iso-exposed WT mice. This may be interpreted as CB2R deficiency alone not being able to induce the activation of microglia, while it enhanced Iso-induced microglial activation and spatial cognitive impairment in the MWM test. These results are consistent with those of previous studies. Nent et al (37) demonstrated that hyperreactive neuropathic pain responses in CB2R KO mice were associated with increased immunostaining of microglial marker Iba1 in the spinal cord. Amenta et al (38) indicated that blockade or deletion of CB2Rs expanded the post-traumatic inflammatory responses in controlled cortical impact model mice, as evidenced by substantial upregulation of iNOS expression and aberrant activation of resident microglia. Sun et al (17) reported that selective activation of CB2R effectively attenuated Iso/surgery-induced hippocampal memory loss in adult mice by downregulating microglia-associated CD11b expression and proinflammatory factors in the hippocampus and medial prefrontal cortex. In an experimental germinal matrix hemorrhage rat model, activation of CB2R by JWH133 downregulated neuroinflammation by promoting microglial M1-to-M2 polarization through the cyclic adenosine monophosphate (cAMP)/protein kinase A (PKA) signaling pathway (39). The underlying relationship of CB2R deletion, the cAMP/PKA pathway and microglial polarization in the pathological process of POCD requires validation in further experiments. All of these results highlight that the neuroprotective effects of $\mathrm{CB} 2 \mathrm{R}$ were associated with the modulation of microglial neuroinflammation in POCD.

In addition to neuroinflammation, neurogenesis and neuroplasticity are considered crucial mechanisms for the endogenous cannabinoid system to exert regulatory roles in numerous neurocognitive disorders (40). CB2R has been linked to the regulation of adult neurogenesis in the mammalian brain. The largest rates of neurogenesis were observed in the subgranular zone of hippocampal DG and in the subventricular zone (41). Thus, in the present study, BrdU immunostaining was performed to detect the neurogenesis ability in hippocampal DG. The results indicated that CB2R deficiency did not alter $\mathrm{BrdU}^{+}$neuronal populations in the hippocampus compared to control mice but significantly enhanced the suppression of neurogenesis induced by Iso exposure. This is in accordance with the results of previous studies. Mensching et al (41) determined that adult CB2R-deficient mice had a stable level 
of proliferation in the hippocampus. Bravo-Ferrer et al (19) revealed that blockade and deletion of $\mathrm{CB} 2 \mathrm{R}$ deteriorated the number of new neurons in the peri-infarct cortex after stroke.

Dendritic spines are post-synaptic structures at a majority of excitatory synapses in the mammalian brain. The number and size of dendritic spines are closely related to cognitive function in different neurological diseases (42). Iso exposure was reported to lead to neuroplastic deficit. Neonatal Iso exposure was indicated to contribute to the decline of dendritic spine densities in the hippocampal DG of juvenile mice accompanied by cognitive deficits in an object recognition test (43). Thus, Golgi staining was performed to detect the dendritic spine destiny in hippocampal DG. In other studies, deletion of CB2R led to alterations of various targets involved in the hippocampal neuroplasticity of mice, including synaptophysin, synaptic transmission, LTP function and dendritic complexity $(22,44)$. However, the present results indicated no difference in spine density between CB2R WT and CB2R KO mice, although the changes after Iso exposure were significantly different. It is possible that CB2R deficiency may be more relevant in feeble conditions in which neurogenesis and neuroplasticity are severely impaired by Iso or traumatic injury.

There are certain limitations to the present study. RT-qPCR experiments were performed to explore the impact of CB2R deficiency on microglial phenotype changes in Iso-induced POCD mice based on a previous study by our group (15). It may be better to detect the expression of M1/M2 microglial phenotypes markers through different tests, including western blot or flow cytometry.

In conclusion, the present study indicated that CB2R deficiency aggravated spatial cognitive impairment in the Iso-induced POCD mouse model. This was partly explained by the aggravated neuroinflammatory reactivity of microglia and enhanced injury to neurogenesis and neuroplasticity in the hippocampus. These results further suggest that $C B 2 R$ is a promising pharmacological target for Iso-induced POCD; however, further research is required to demonstrate its validity.

\section{Acknowledgements}

Not applicable.

\section{Funding}

No funding was received.

\section{Availability of data and materials}

The datasets used and/or analyzed during the current study are available from the corresponding author on reasonable request.

\section{Authors' contributions}

CL and JPS were responsible for designing the study, performing the experiment, collecting the data and writing the manuscript. JGS and YS were responsible for designing the study, performing the experiment, and collecting the data and reviewing the manuscript. HJ was responsible for providing experimental ideas and reviewing the manuscript. CL and HJ were responsible for the confirming the authenticity of all the raw data. All authors read and approved the final manuscript.

\section{Ethics approval and consent to participate}

All experiments were performed with the approval of the Animal Care Committee of the Fourth Hospital of Hebei Medical University (Shijiazhuang, China).

\section{Patient consent for publication}

Not applicable.

\section{Competing interests}

The authors declare that they have no competing interests.

\section{References}

1. Evered LA and Silbert BS: Postoperative cognitive dysfunction and noncardiac surgery. Anesth Analg 127: 496-505, 2018.

2. Hovens IB, Schoemaker RG, van der Zee EA, Heineman E, Izaks GJ and van Leeuwen BL: Thinking through postoperative cognitive dysfunction: How to bridge the gap between clinical and pre-clinical perspectives. Brain Behav Immun 26: 1169-1179, 2012.

3. Rundshagen I: Postoperative cognitive dysfunction. Dtsch Arztebl Int 111: 119-125, 2014.

4. Monk TG, Weldon BC, Garvan CW, Dede DE, van der Aa MT, Heilman KM and Gravenstein JS: Predictors of cognitive dysfunction after major noncardiac surgery. Anesthesiology 108: 18-30, 2008.

5. Kapila AK, Watts HR, Wang T and Ma D: The impact of surgery and anesthesia on post-operative cognitive decline and Alzheimer's disease development: Biomarkers and preventive strategies. J Alzheimers Dis 41: 1-13, 2014.

6. Spangenberg EE and Green KN: Inflammation in Alzheimer's disease: Lessons learned from microglia-depletion models. Brain Behav Immun 61: 1-11, 2017.

7. Sung PS, Lin PY, Liu CH, Su HC and Tsai KJ: Neuroinflammation and neurogenesis in Alzheimer's disease and potential therapeutic approaches. Int J Mol Sci 21: 21, 2020.

8. Skvarc DR, Berk M, Byrne LK, Dean OM, Dodd S, Lewis M, Marriott A, Moore EM, Morris G, Page RS, et al: Post-operative cognitive dysfunction: An exploration of the inflammatory hypothesis and novel therapies. Neurosci Biobehav Rev 84: 116-133, 2018.

9. Safavynia SA and Goldstein PA: The Role of neuroinflammation in postoperative cognitive dysfunction: Moving from hypothesis to treatment. Front Psychiatry 9: 752, 2019.

10. Tang Y and Le W: Differential roles of M1 and M2 microglia in neurodegenerative diseases. Mol Neurobiol 53: 1181-1194, 2016.

11. Santos LE, Beckman D and Ferreira ST: Microglial dysfunction connects depression and Alzheimer's disease. Brain Behav Immun 55: 151-165, 2016.

12. Komorowska-Müller JA and Schmöle AC: CB2 Receptor in microglia: The guardian of self-control. Int J Mol Sci 22: 19, 2020.

13. Aso E and Ferrer I: CB2 Cannabinoid receptor as potential target against Alzheimer's disease. Front Neurosci 10: 243, 2016.

14. Basavarajappa BS, Shivakumar M, Joshi V and Subbanna S: Endocannabinoid system in neurodegenerative disorders. J Neurochem 142: 624-648, 2017.

15. Li C, Shi J, Wang B, Li J and Jia H: CB2 cannabinoid receptor agonist ameliorates novel object recognition but not spatial memory in transgenic APP/PS1 mice. Neurosci Lett 707: 134286, 2019.

16. Schmöle AC, Lundt R, Toporowski G, Hansen JN, Beins E, Halle A and Zimmer A: Cannabinoid receptor 2-deficiency ameliorates disease symptoms in a mouse model with Alzheimer's diseaselike pathology. J Alzheimers Dis 64: 379-392, 2018. 
17. Sun L, Dong R, Xu X, Yang X and Peng M: Activation of cannabinoid receptor type 2 attenuates surgery-induced cognitive impairment in mice through anti-inflammatory activity. J Neuroinflammation 14: 138, 2017.

18. Taupin P: BrdU immunohistochemistry for studying adult neurogenesis: Paradigms, pitfalls, limitations, and validation. Brain Res Brain Res Rev 53: 198-214, 2007.

19. Bravo-Ferrer I, Cuartero MI, Zarruk JG, Pradillo JM, Hurtado O, Romera VG, Díaz-Alonso J, García-Segura JM, Guzmán M, Lizasoain I, et al: Cannabinoid Type-2 receptor drives neurogenesis and improves functional outcome after stroke. Stroke 48: 204-212, 2017.

20. Mancuso JJ, Chen Y, Li X, Xue Z and Wong ST: Methods of dendritic spine detection: From Golgi to high-resolution optical imaging. Neuroscience 251: 129-140, 2013.

21. Jiang Y, Liu Y, Zhu C, Ma X, Ma L, Zhou L, Huang Q, Cen L, $\mathrm{Pi} R$ and Chen X: Minocycline enhances hippocampal memory, neuroplasticity and synapse-associated proteins in aged $\mathrm{C} 57$ BL/6 mice. Neurobiol Learn Mem 121: 20-29, 2015.

22. Li Y and Kim J: Deletion of CB2 cannabinoid receptors reduces synaptic transmission and long-term potentiation in the mouse hippocampus. Hippocampus 26: 275-281, 2016.

23. Li Y and Kim J: CB2 Cannabinoid receptor knockout in mice impairs contextual long-term memory and enhances spatial working memory. Neural Plast 2016: 9817089, 2016.

24. Liu J, Wang P, Zhang X, Zhang W and Gu G: Effects of different concentration and duration time of isoflurane on acute and long-term neurocognitive function of young adult C57BL/6 mouse. Int J Clin Exp Pathol 7: 5828-5836, 2014.

25. Vorhees CV and Williams MT: Morris water maze: Procedures for assessing spatial and related forms of learning and memory. Nat Protoc 1: 848-858, 2006.

26. Schmöle AC,Lundt R, Ternes S, Albayram Ö, Ulas T, Schultze JL, Bano D, Nicotera P, Alferink J and Zimmer A: Cannabinoid receptor 2 deficiency results in reduced neuroinflammation in an Alzheimer's disease mouse model. Neurobiol Aging 36: 710-719, 2015.

27. Livak KJ and Schmittgen TD: Analysis of relative gene expression data using real-time quantitative PCR and the 2(-Delta Delta C(T)) Method. Methods 25: 402-408, 2001.

28. Chen B, Bromley-Brits K, He G, Cai F, Zhang X and Song W: Effect of synthetic cannabinoid HU210 on memory deficits and neuropathology in Alzheimer's disease mouse model. Curr Alzheimer Res 7: 255-261, 2010.

29. Zöller T, Attaai A, Potru PS, Ruß T and Spittau B: Aged Mouse cortical microglia display an activation profile suggesting immunotolerogenic functions. Int J Mol Sci 19: 19, 2018.

30. Hem S, Albite R, Loresi M, Rasmussen J, Ajler P, Yampolsky C, Chabot JD, Gerszten PC and Goldschmidt E: Pathological changes of the hippocampus and cognitive dysfunction following frontal lobe surgery in a rat model. Acta Neurochir (Wien) 158 2163-2171, 2016.

31. Qiu LL, Pan W, Luo D, Zhang GF, Zhou ZQ, Sun XY, Yang JJ and Ji MH: Dysregulation of BDNF/TrkB signaling mediated by NMDAR/Ca $2^{+} /$calpain might contribute to postoperative cognitive dysfunction in aging mice. J Neuroinflammation 17 : $23,2020$.
32. Contino M, Capparelli E, Colabufo NA and Bush AI: Editorial: The CB2 cannabinoid system: A new strategy in neurodegenerative disorder and neuroinflammation. Front Neurosci 11: 196, 2017.

33. Bromley-Brits K, Deng Y and Song W: Morris water maze test for learning and memory deficits in Alzheimer's disease model mice. J Vis Exp 53: 2920, 2011.

34. Aso E, Andrés-Benito P, Carmona M, Maldonado R and Ferrer I: Cannabinoid receptor 2 participates in amyloid- $\beta$ processing in a mouse model of Alzheimer's disease but plays a minor role in the therapeutic properties of a cannabis-based medicine. J Alzheimers Dis 51: 489-500, 2016.

35. Feng X, Valdearcos M, Uchida Y, Lutrin D, Maze M and Koliwad SK: Microglia mediate postoperative hippocampal inflammation and cognitive decline in mice. JCI Insight 2: e91229, 2017.

36. Wang HL, Liu H, Xue ZG, Liao QW and Fang H: Minocycline attenuates post-operative cognitive impairment in aged mice by inhibiting microglia activation. J Cell Mol Med 20: 1632-1639, 2016.

37. Nent E, Nozaki C, Schmöle AC, Otte D and Zimmer A: CB2 receptor deletion on myeloid cells enhanced mechanical allodynia in a mouse model of neuropathic pain. Sci Rep 9: 7468, 2019.

38. Amenta PS, Jallo JI, Tuma RF, Hooper DC and Elliott MB: Cannabinoid receptor type-2 stimulation, blockade, and deletion alter the vascular inflammatory responses to traumatic brain injury. J Neuroinflammation 11: 191, 2014.

39. Tao Y, Li L, Jiang B, Feng Z, Yang L, Tang J, Chen Q, Zhang J, Tan Q, Feng H, et al: Cannabinoid receptor-2 stimulation suppresses neuroinflammation by regulating microglial M1/M2 polarization through the cAMP/PKA pathway in an experimental GMH rat model. Brain Behav Immun 58: 118-129, 2016.

40. Cassano T, Calcagnini S, Pace L, De Marco F, Romano A and Gaetani S: Cannabinoid receptor 2 signaling in neurodegenerative disorders: From pathogenesis to a promising therapeutic target. Front Neurosci 11: 30, 2017.

41. Mensching L, Djogo N, Keller C, Rading S and Karsak M: Stable adult hippocampal neurogenesis in cannabinoid receptor CB2 deficient mice. Int J Mol Sci 20: 20, 2019.

42. Zhang K, Lian N, Ding R, Guo C, Dong X, Li Y, Wei S, Jiao Q, Yu Y and Shen H: Sleep deprivation aggravates cognitive impairment by the alteration of hippocampal neuronal activity and the density of dendritic spine in isoflurane-exposed mice. Front Behav Neurosci 14: 589176, 2020.

43. Schaefer ML, Perez PJ, Wang M, Gray C, Krall C, Sun X, Hunter E, Skinner J and Johns RA: Neonatal isoflurane anesthesia or disruption of postsynaptic density-95 protein interactions change dendritic spine densities and cognitive function in juvenile mice. Anesthesiology 133: 812-823, 2020.

44. García-Gutiérrez MS, Ortega-Álvaro A, Busquets-García A, Pérez-Ortiz JM, Caltana L, Ricatti MJ, Brusco A, Maldonado R and Manzanares J: Synaptic plasticity alterations associated with memory impairment induced by deletion of CB2 cannabinoid receptors. Neuropharmacology 73: 388-396, 2013.

This work is licensed under a Creative Commons Attribution-NonCommercial-NoDerivatives 4.0 International (CC BY-NC-ND 4.0) License. 